\title{
SOLID PARTICLE CHARACTERIZATION. SOME ASPECT RELATED TO THE SHAPE EVALUATION BY USING SOFTWARE CORRELATIONS
}

\author{
EMILIAN-FLORIN MOSNEGUTU ${ }^{1}$, VALENTIN NEDEFF ${ }^{1,2}$, ALEXANDRA- \\ DANA CHITIMUS ${ }^{* 1}$, MARCIN JASIŃSKI ${ }^{3}$, NARCIS BARSAN ${ }^{1}$ \\ 1 "Vasile Alecsandri" University of Bacau, Calea Marasesti 157, Bacau, 600115, \\ Romania \\ ${ }^{2}$ The Academy of Agricultural and Forestry Sciences "Gheorghe Ionescu, Sisesti, Bucharest, \\ Romania \\ ${ }^{3}$ Institute of Vehicles, Warsaw University of Technology, Poland
}

\begin{abstract}
This article presents a method for determining the Riley circularity evaluation, a characteristic that defines the shape of a solid particle. To determine this parameter, a working methodology was identified by using Mathcad. To verify this methodology 15 anthracite particles were inserted in the working file, the shape of which is different, to determine the analyzed characteristic.
\end{abstract}

Keywords: riley's circularity, Mathcad 15, solid particle

\section{INTRODUCERE}

In the industrial processes, raw materials are used (in the case of the milling and bakery industry, the pharmaceutical industry, the sugar products industry etc.) whose size and shape vary greatly. And in some technological processes, products of different sizes and shapes can be obtained (the process of crushing or granulation). Identifying the dimensional elements of a solid particle, especially if they have at less than $1 \mathrm{~cm}$ in size, can sometimes become a challenge.

Also identifying the shape of a real solid particle is a challenge and there is no well-defined method. Such simple definitions are not sufficiently precise to evaluate different methods of measuring particle size or to incorporate them as parameters in the equations in which the solid particle form is a calculation parameter [1-3].

Some researches were attempts to identify different methods for classifying the shapes of solid particles, which is why the terminology has been enriched with a series of terms such as roundness or sphericity etc., but unfortunately, even today, there is no some general approach in these domain [4-10].

Over the years, several studies introduced some methodologies to measure particle shape. For the dimensional determination of solid particles, the classical method is generally used, respectively the dimensional determination using the binder, a rather improbable method, which is why a series of visual graphical representations have been designed, to reduce the measurement time [11-18]. Dimensional determination using the binder was introduced to determine the flattening index or elongation but is limited only to certain particle sizes, for practical reasons [19].

\footnotetext{
* Corresponding author, email: dana.chitimus@ub.ro

(C) 2021 Alma Mater Publishing House
} 
Recently, computer-based image analysis has been applied to dimensional research [20-22], thus obtaining practical methods for determining the particle size with good results [20,21]. Determining the shape of particles through the computer helps a lot and reduce the measurement time [3,23]. According to the literature, a series of terms can be used to identify the shape of a solid particle, and for the same term are use a different calculation relation.

One of the characteristics that describe the shape of a solid particle is Riley's circularity. To determine it, must determine the values of the diameters of two circles, the inscribed circle, and the circle circumscribed to the studied particle must be known (Figure 1) and can be determined with the equation:

$$
\Phi_{S R}=\sqrt{\frac{2 \cdot R_{i}}{2 \cdot R_{C}}}
$$

were $R_{i}$ represent the radius of the inscribed circle, $R_{c}$ - radius of the circumscribed circle.

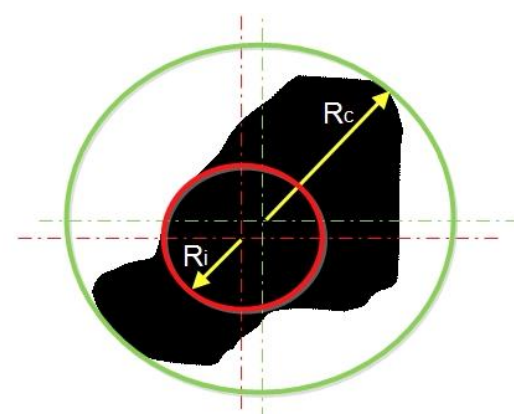

Fig. Error! No text of specified style in document.1. Determining Riley's circularity.

This article presents the calculation methodology, using the Mathcad 15 program, to determine the Riley circularity.

\section{EXPERIMENTAL SETUP}

\subsection{Equipment and materials}

To determine the Riley circularity of different types of solid particles, used as granular filter material, for example, the first stage meant creating individual pictures using a microscope, with an optical zoom of 4 . The material used in this study was anthracite particles obtained as refuse on the $2 \mathrm{~mm}$ sieve. The methodology, for determining the Riley circularity of anthracite solid particles (Figure 1), was applied to individual 15 solid particles.

\subsection{Working methodology}

This article presents the working methodology (Figure 2) for determining the Riley circularity of particles with millimeter dimensions using a work program. All work operations, image reading, and data processing was performed by using the Mathcad 15 program [24].

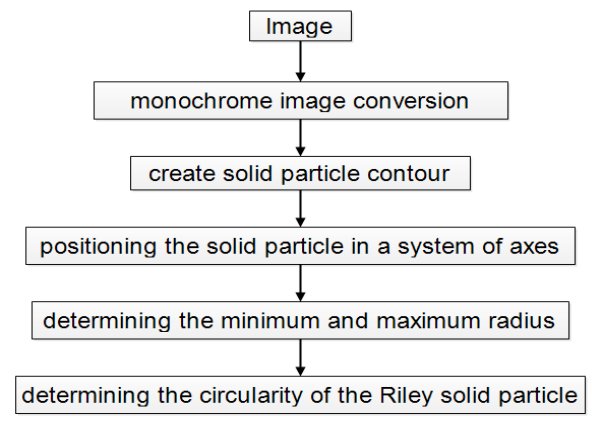

Fig. 2. Working methodology. 
This method involves using the Mathcad 15 program to process the image to determine Riley's circularity. The work steps for determining Riley's circularity were:

- The image, corresponding to the solid particle (Figure 3), was uploaded in the working file, following that, to be processed, it was converted from color image to monochromatic image with a design to make the matrix corresponding to the image into a matrix within to which only two numbers 0 and 255 are inserted.

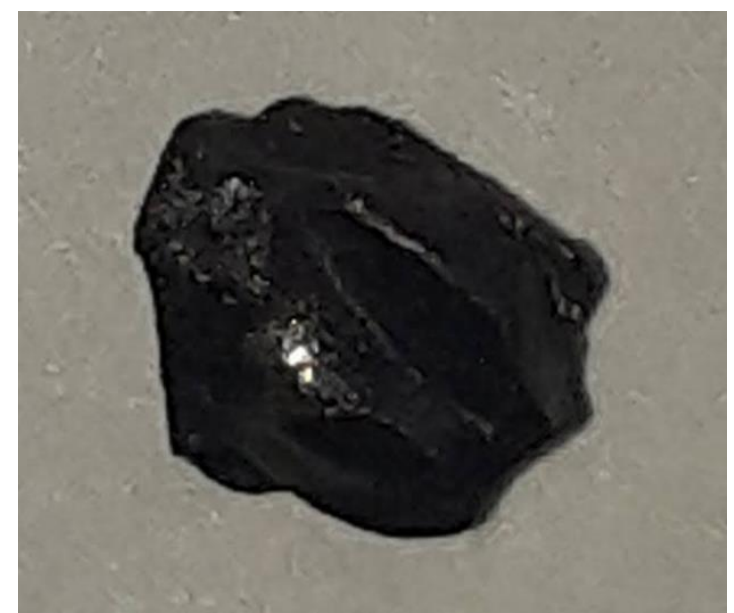

a)

\begin{tabular}{|l|l|l|l|l|l|l|l|l|l|l|}
\hline & 0 & 1 & 2 & 3 & 4 & 5 & 6 & 7 & 8 & 9 \\
\hline 0 & 150 & 150 & 150 & 150 & 150 & 150 & 149 & 149 & 148 & 148 \\
\hline 1 & 150 & 150 & 150 & 150 & 150 & 151 & 150 & 149 & 149 & 149 \\
\hline 2 & 151 & 151 & 151 & 151 & 151 & 151 & 150 & 150 & 149 & 149 \\
\hline 3 & 151 & 151 & 151 & 151 & 151 & 151 & 151 & 150 & 150 & 148 \\
\hline 4 & 152 & 152 & 152 & 152 & 152 & 151 & 151 & 151 & 150 & 151 \\
\hline 5 & 152 & 152 & 152 & 152 & 152 & 151 & 151 & 151 & 150 & 150 \\
\hline 6 & 151 & 152 & 152 & 152 & 152 & 151 & 151 & 151 & 150 & 150 \\
\hline 7 & 151 & 152 & 152 & 153 & 153 & 151 & 151 & 151 & 150 & 150 \\
\hline 8 & 151 & 152 & 153 & 153 & 152 & 151 & 151 & 150 & 150 & 151 \\
\hline 9 & 152 & 153 & 153 & 153 & 152 & 151 & 151 & 151 & 150 & 150 \\
\hline 10 & 152 & 153 & 154 & 154 & 152 & 151 & 151 & 151 & 150 & 150 \\
\hline 11 & 153 & 153 & 154 & 154 & 152 & 151 & 151 & 151 & 150 & 150 \\
\hline 12 & 153 & 154 & 154 & 155 & 152 & 151 & 151 & 151 & 150 & 150 \\
\hline 13 & 153 & 154 & 155 & 155 & 152 & 151 & 151 & 151 & 150 & 150 \\
\hline 14 & 151 & 152 & 152 & 152 & 150 & 150 & 150 & 150 & 150 & 150 \\
\hline 15 & 151 & 152 & 151 & 152 & 151 & 151 & 151 & 151 & 151 & $\ldots$ \\
\hline
\end{tabular}

b)

Fig. 3. Presentation of the working image: a) the image used; b) the matrix corresponding to the image.

For that the following functions can be used to make this change, from color image to the monochrome image: a. function through which the values within the working matrix can be changed:

$$
f(x):=\text { if }(x>a, 255,0)
$$

wherein $a$ represents a randomly chosen value, depending in particular on the color spectrum of the image under analysis, and $x$ represents the matrix subject to transformation (in Figure 1.b it is noted by $a l$ ).

b. or using the function in the Mathcad 15 program "binarize_auto", the function that converts the color image into a black and white image.

The processing method will be chosen according to the colors contained in the processed image. In the end, of this type of processing, the image obtained is in the form of that shown in Figure 4.

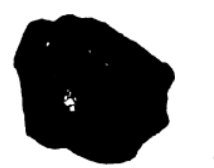

\begin{tabular}{|c|l|l|l|l|l|l|l|l|l|r|}
\hline & 0 & 1 & 2 & 3 & 4 & 5 & 6 & 7 & 8 & 9 \\
\hline 0 & 255 & 255 & 255 & 255 & 255 & 255 & 255 & 255 & 255 & 255 \\
\hline 1 & 255 & 255 & 255 & 255 & 255 & 255 & 255 & 255 & 255 & 255 \\
\hline 2 & 255 & 255 & 255 & 255 & 255 & 255 & 255 & 255 & 255 & 255 \\
\hline 3 & 255 & 255 & 255 & 255 & 255 & 255 & 255 & 255 & 255 & 255 \\
\hline 4 & 255 & 255 & 255 & 255 & 255 & 255 & 255 & 255 & 255 & 255 \\
\hline 5 & 255 & 255 & 255 & 255 & 255 & 255 & 255 & 255 & 255 & 255 \\
\hline 6 & 255 & 255 & 255 & 255 & 255 & 255 & 255 & 255 & 255 & 255 \\
\hline 7 & 255 & 255 & 255 & 255 & 255 & 255 & 255 & 255 & 255 & 255 \\
\hline 8 & 255 & 255 & 255 & 255 & 255 & 255 & 255 & 255 & 255 & 255 \\
\hline 9 & 255 & 255 & 255 & 255 & 255 & 255 & 255 & 255 & 255 & 255 \\
\hline 10 & 255 & 255 & 255 & 255 & 255 & 255 & 255 & 255 & 255 & 255 \\
\hline 11 & 255 & 255 & 255 & 255 & 255 & 255 & 255 & 255 & 255 & 255 \\
\hline 12 & 255 & 255 & 255 & 255 & 255 & 255 & 255 & 255 & 255 & 255 \\
\hline 13 & 255 & 255 & 255 & 255 & 255 & 255 & 255 & 255 & 255 & 255 \\
\hline 14 & 255 & 255 & 255 & 255 & 255 & 255 & 255 & 255 & 255 & 255 \\
\hline 15 & 255 & 255 & 255 & 255 & 255 & 255 & 255 & 255 & 255 & $\ldots$ \\
\hline
\end{tabular}

a)

b)

Fig. 4. The studied particle: a) monochromatic image; b) the matrix corresponding to the studied image.

The analysis of this picture shows that in addition to the image of the solid particle other external elements are not components of the solid particle, elements that must be automatically removed from the image. For this purpose, the Mathcad 15 program canny function was used, that generating an image through which only the contour of the 
studied particle is highlighted. This processing results in the following image that will be further worked on (Figure $5)$.

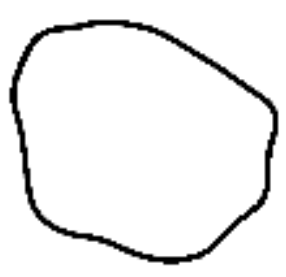

Fig. 5. Solid particle contour.

The next stage of image processing is its framing in a system of axes whose center corresponds to the center of the analyzed particle. For this, the following calculation conventions were performed:

a. A matrix is defined:

$$
b=\operatorname{match}(0, a)
$$

where match represents a function in Mathcad 15, function that has the role of looking in the working matrix a defined value, in our case 0 (because the value 0 corresponds to the color black, color with which the solid particle is highlighted, and with white color, respectively with value 255 , in the background highlight). The matrix subjected to coding is subsequently optimized and in the obtained result are presented the indices of each value obtained. The term $a$ represent the matrix under analysis and defines the image under analysis.

b. The matrix thus obtained is processed to define the coordinates of a point to achieve the graphical representation of the image under analysis:

$$
x_{k}=\left(b_{k}\right)_{1} \quad y_{k}=\left(b_{k}\right)_{0}
$$

in which: $\mathrm{k}$ represents a range of values between $0 \ldots . \mathrm{N}$, where $\mathrm{N}$ is defined as a number given by the relation:

$$
\mathrm{N}=\operatorname{rows}(\mathrm{b})-1
$$

and depends on the number of rows in the matrix $b$.

- To frame the studied particle in the center of a coordinate system, the following calculation artifice is made:

$$
x 1=x-\operatorname{mean}(x) \quad y 1=y-\operatorname{mean}(y)
$$

in which the mean function generates the average value of the group of values corresponding to the parameter $x$ or $y$.

Following this processing, the result obtained is shown in Figure 6.

To determine the diameter of the inscribed and the circumscribed circle, the values of the diameters corresponding to them must be known. For this purpose, the largest and smallest distance from the center of the axis system to the particle contour will be determined, respectively the radius of the circumscribed circle and the inscribed circle will be obtained (Figure 7). When determining the value of these two parameters, the relationship of calculating the distance between two points will be used, the first point being with the values for the $O X$ axis equal to 0 , as well as for the $O Y$ axis.

$$
\Delta=\sqrt{(0-x)^{2}+(0-y)^{2}}
$$

But the values of the radius of the inscribed circle, respectively circumscribed circle, are much too large reason for which, individually, for the inscribed circle and the circumscribed circle, an adjustment of the center of the circle will be made. 


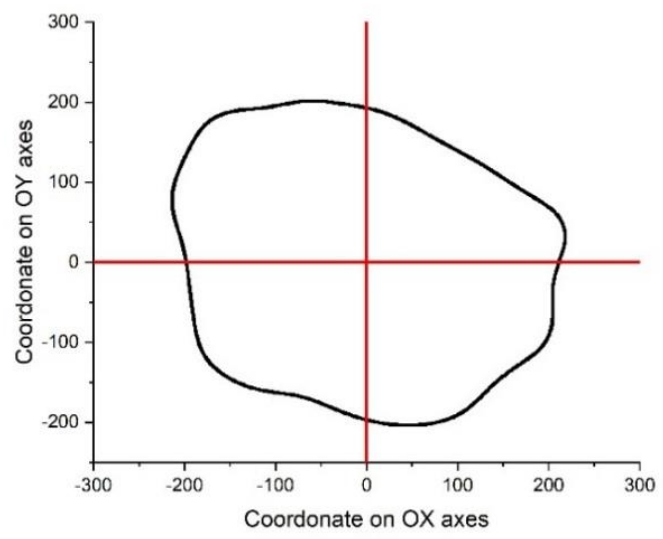

Fig. 6. Framing the solid particle contour in a coordinate system.

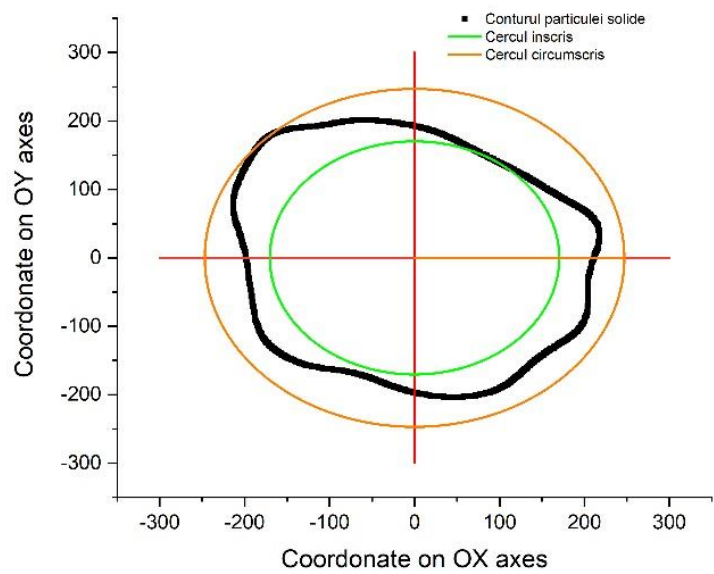

Fig. 7. Presentation of the two circles.

For this purpose, the points of intersection between the contour of the solid particle and the inscribed circle and circumscription circle will be identified, the reason for which the following working steps has been made:

- a new matrix was created that includes the values of $\Delta, x$ and $y$ using the function:

$$
D 1:=\text { augment }(\Delta, x, y)
$$

- was define the three columns of the newly created matrix:

$$
d d:=D 1^{<0>} ; x x:=D 1^{<1>} ; y y:=D 1^{<2>}
$$

- was identify the coordinates of the minimum and maximum point of the parameter $d d$ ( $d d$ being given by the distance between the center of the axis system and the contour of the analyzed particle):

$$
\begin{aligned}
& \left\{\begin{array}{l}
\operatorname{amx}:=\operatorname{lookup}(\min (d d), d d, x x) \\
\operatorname{amy}:=\operatorname{lookup}(\min (d d), d d, y y)
\end{array}\right. \\
& \left\{\begin{array}{l}
a M x:=\operatorname{lookup}(\max (d d), d d, x x) \\
\operatorname{aMx}:=\text { lookup }(\max (d d), d d, y y)
\end{array}\right.
\end{aligned}
$$


The value of the angle corresponding to the obtained coordinates, concerning the value of 0 degrees is determined with the help of the following programming functions:

$$
\begin{aligned}
& \propto 1:=\left\{\begin{array}{c}
\left(\operatorname{atan}\left(\frac{|a m x|}{|a m y|}\right)\right) \cdot \frac{180}{\pi} \text { if }|a m x|>0 \wedge \mid \text { amy } \mid>0 \\
\left(\operatorname{atan}\left(\frac{|a m x|}{|a m y|}\right)\right) \cdot \frac{180}{\pi}+90 \text { if }|a m x|<0 \wedge|a m y|>0 \\
\left(\operatorname{atan}\left(\frac{|a m x|}{|a m y|}\right)\right) \cdot \frac{180}{\pi}+180 \text { if }|a m x|<0 \wedge|a m y|<0 \\
\left(\operatorname{atan}\left(\frac{|a m x|}{|a m y|}\right)\right) \cdot \frac{180}{\pi}+270 \text { if }|a m x|>0 \wedge|a m y|<0
\end{array}\right. \\
& \alpha 2:=\left\{\begin{array}{l}
\left(\operatorname{atan}\left(\frac{|a M x|}{|a M y|}\right)\right) \cdot \frac{180}{\pi} \text { if }|a M x|>0 \wedge|a M y|>0 \\
\left(\operatorname{atan}\left(\frac{|a M x|}{|a M y|}\right)\right) \cdot \frac{180}{\pi}+90 \text { if }|a M x|<0 \wedge|a M y|>0 \\
\left(\operatorname{atan}\left(\frac{|a M x|}{|a M y|}\right)\right) \cdot \frac{180}{\pi}+180 \text { if }|a M x|<0 \wedge|a M y|<0 \\
\left(\operatorname{atan}\left(\frac{|a M x|}{|a M y|}\right)\right) \cdot \frac{180}{\pi}+270 \text { if }|a M x|>0 \wedge|a M y|<0
\end{array}\right.
\end{aligned}
$$

where $\alpha l$ has noted the angle obtained with the horizontal and the point of intersection of the inscribed circle and $\alpha 2$ the angle obtained with the horizontal and the point of intersection of the circumscribed circle. Thus, the value of the angle can be identified.

Opposite the point of intersection between the contour of the particle and the circumscribed circle and the inscribed circle, there is a space that must be partially removed. The value of the angle corresponding to the respective space is determined with the calculation relation:

$$
\left\{\begin{array}{l}
\alpha 11=\alpha 1+180 \\
\alpha 21=\alpha 2+180
\end{array}\right.
$$

At this point, no correlation can be made between these two values of the angle with coordinates corresponding to that point. Therefore, the angular value corresponding to all points corresponding to the contour of the solid particle was identified:

$$
\alpha 3_{k}:=\left\{\begin{array}{l}
\left(\operatorname{atan}\left(\frac{\left|x_{k}\right|}{\left|y_{k}\right|}\right)\right) \cdot \frac{180}{\pi} \text { if } x_{k}>0 \wedge y_{k}>0 \\
\left(\operatorname{atan}\left(\frac{\left|x_{k}\right|}{\left|y_{k}\right|}\right)\right) \cdot \frac{180}{\pi}+90 \text { if } x_{k}<0 \wedge y_{k}>0 \\
\left(\operatorname{atan}\left(\frac{\left|x_{k}\right|}{\left|y_{k}\right|}\right)\right) \cdot \frac{180}{\pi}+180 \text { if } x_{k}<0 \wedge y_{k}<0 \\
\left(\operatorname{atan}\left(\frac{\left|x_{k}\right|}{\left|y_{k}\right|}\right)\right) \cdot \frac{180}{\pi}+270 \text { if } x_{k}>0 \wedge y_{k}<0
\end{array}\right.
$$

the value that is entered in a second matrix that also includes the coordinates of the contour of the studied particle $(x$ and $y)$

$$
k_{k}:=\operatorname{augment}(\alpha 3, x, y)
$$

and it defines the three columns of the newly created matrix as follows: 


$$
d d 2:=k^{<0>} ; x x 2:=k^{<1>} ; y y 2:=k^{<2>}
$$

The values of the angles $\alpha 11$ and $\alpha 31$ (defined in the calculation relations (10)) in the human situations are not found in the series of values corresponding to $\alpha 2$, the reason for which the following calculation artifice is realized: - Subtract from the value of the angle corresponding to the points using which the contour of the analyzed particle is drawn the value of the angle opposite to the intersection between the particle account and the two circles. Because, in some cases, they are values lower than the value of the angle opposite to the intersection angle this difference is modulated:

$$
\alpha 12_{k}:=\left|\alpha 3_{k}-\alpha 1\right| \quad \alpha 22_{k}:=\left|\alpha 2_{k}-\alpha 3\right|
$$

- Identify the value of the angle opposite to the angle corresponding to the intersection between the contour of the particle and the inscribed and circumscribed circle:

$$
\alpha 13:=\alpha 11 \pm \min (\alpha 12) \quad \alpha 23:=\alpha 21 \pm \min (\alpha 22)
$$

As a result of this artifice, the coordinates opposite the intersection points can be identified:

$$
\begin{aligned}
& \operatorname{amx} 1:=\operatorname{lookup}(\alpha 13, d d 2, x x 2) \\
& \operatorname{amy} 1:=\operatorname{lookup}(\alpha 13, d d 2, y y 2) \\
& \operatorname{aMx1}:=\operatorname{lookup}(\alpha 23, d d 2, x x 2) \\
& \operatorname{aMx} 1:=\operatorname{lookup}(\alpha 23, d d 2, y y 2)
\end{aligned}
$$

Knowing the coordinates of the respective points can determine the distance between the center of the current system of axes and the respective points:

$$
\Delta m 1=\sqrt{(0-a m x 1)^{2}+(0-a m y 1)^{2}} \quad \Delta M 1=\sqrt{(0-a M x 1)^{2}+(0-a M y 1)^{2}}
$$

And now it is possible to determine the value with which the coordinates of the center of the axis system will change, values necessary for translating the center of the axis system so that the two circles include and be included in the studied contour.

$$
\operatorname{dif} 1:=\frac{|r m 1-\Delta m 1|}{2} \quad \text { dif } 2:=\frac{|R m 1-\Delta M 1|}{2}
$$

Therefore it will be determined, for each type of circle a different center of the axis system and another corresponding radius, the following work steps are performed:

- for the inscribed circle:

$$
\begin{gathered}
x 1=x-\operatorname{mean}(x) \pm \operatorname{dif} 1 \quad y 1=y-\operatorname{mean}(y) \pm \operatorname{dif} 1 \\
\Delta m m_{k}=\sqrt{\left(0-x 1_{k}\right)^{2}+\left(0-y 1_{k}\right)^{2}} \\
\operatorname{Rm} 2:=\min (\Delta \mathrm{mm})
\end{gathered}
$$

- for the circumscribed circle:

$$
\begin{gathered}
x M=x-\operatorname{mean}(x) \pm \operatorname{dif} 2 \quad y M=y-\operatorname{mean}(y) \pm \operatorname{dif} 2 \\
\Delta M m_{k}=\sqrt{\left(0-x M_{k}\right)^{2}+\left(0-y M_{k}\right)^{2}} \\
\operatorname{Rm} 2:=\max (\Delta \mathrm{Mm})
\end{gathered}
$$

For the control will be made, only for the circumscribed circle, the next difference from it being made that the maximum value is as close as possible to the value 0 . 


$$
\text { contr }_{k}:=\left|\Delta M m_{k}-\mathrm{Rm} 2\right|
$$

Figure 8 shows both adjustments made.
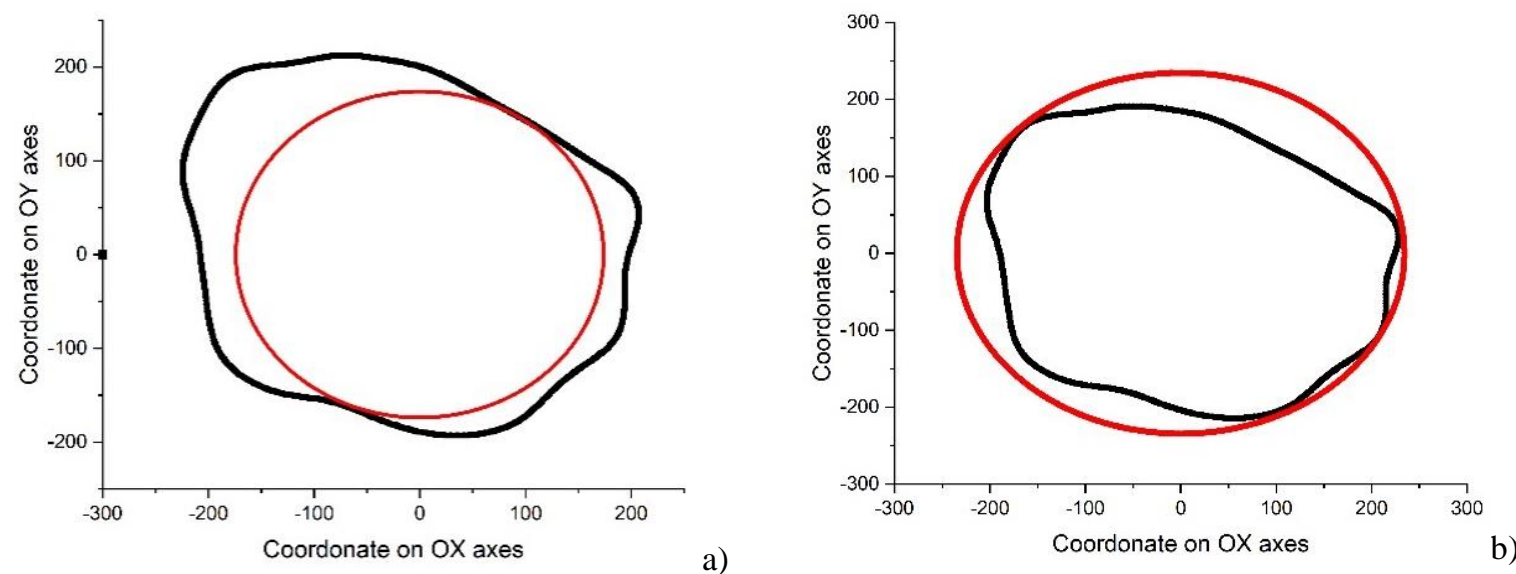

Fig. 8. The new position of the two circles: a) the inscribed circle; b) the circumscribed circle.

The values thus obtained can be used to determine the circularity of Riley to the solid particle using the calculation relation (1).

\section{RESULTS AND DISCUSSION}

The previously presented methodology was used on different solid anthracite particles. In Figure 9 are presented 4 used solid particles.
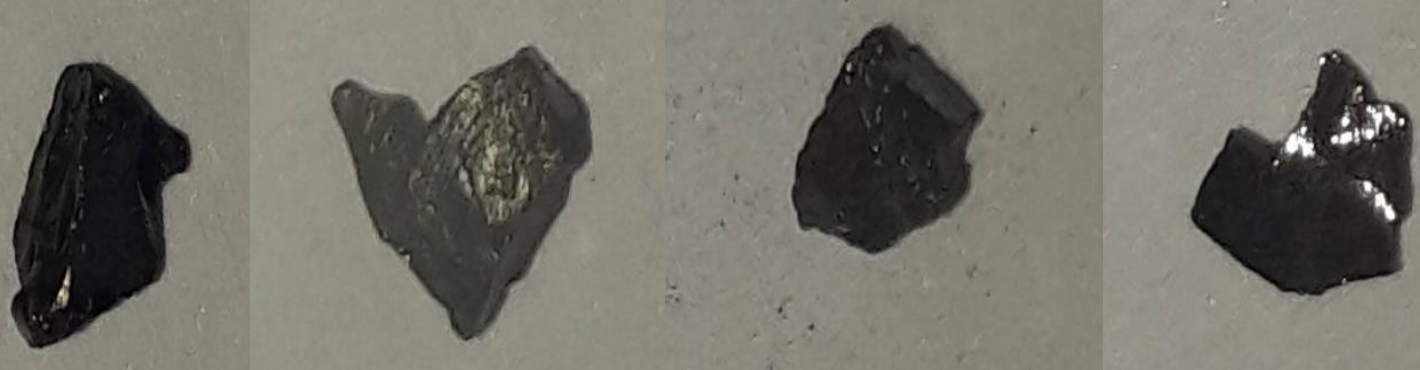

Fig. 9. Example of the used anthracite particles.

After processing the images from Figure 9, it was possible to graphically represent the results obtained, corresponding to the two main stages of identifying the studied parameter (before centering and after centering), respectively Riley's circularity (Figure 10).

Following the analysis of the obtained results, by implementing the methodology presented above for determining the circularity of Riley for 15 solid particles, respectively anthracite particles, it was possible to draw their variation (Figure 11) taking into account the two stages corresponding to the working methodology. 

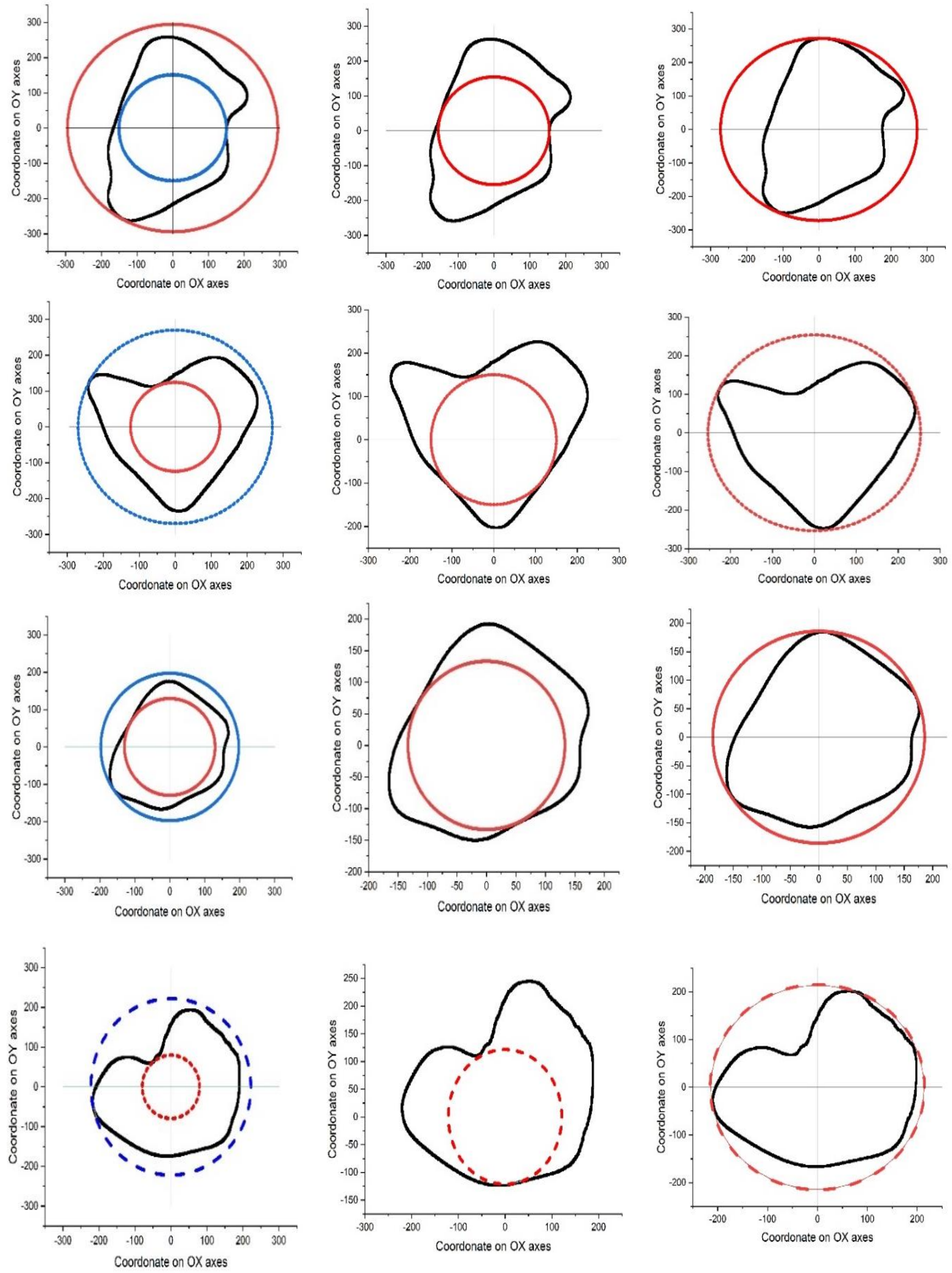

a)

b)

c)

Fig. 10. Graphical representations of the inscribed and circumscribed circle: a) for the initial working situation; b) and c) after the individual centering of the circles.

Analyzing the obtained results, it is found that the largest difference between the values obtained is for particle number 5 , respectively $79.76 \%$, and the smallest difference was obtained for particle number 7 of $99.59 \%$. 


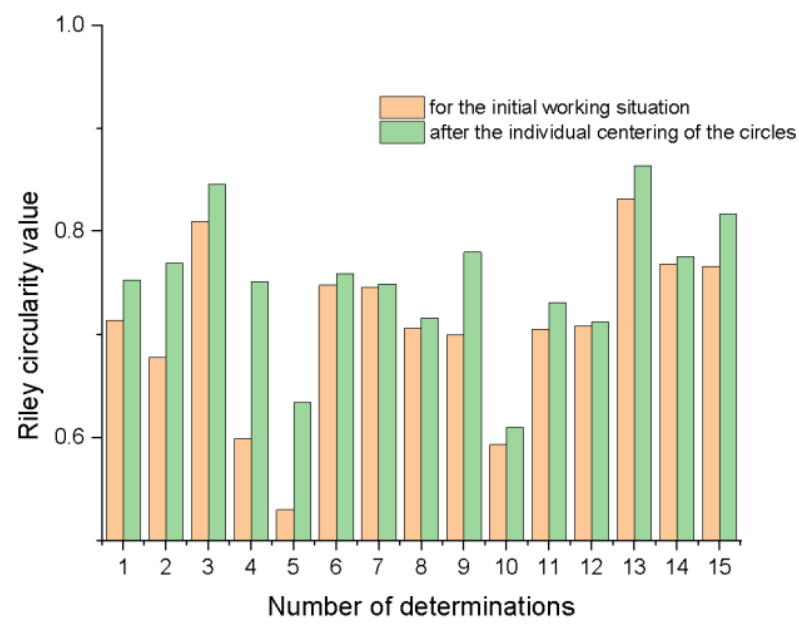

Fig. 11. Variation of the Riley circularity value for different anthracite particles.

\section{CONCLUSIONS}

Following the analysis of the obtained results, the following conclusions can be drawn:

- Products obtained from agriculture but also various industries are characterized by different sizes and shapes;

- The shape of the solid particle is a feature that is used in the design stages of various process equipment;

- Over time, a series of calculation relations have been generated to describe as accurately as possible the shape of a solid particle;

- Riley's circularity is that characteristic of the shape of a solid particle which is described by the ratio between the diameters of the inscribed and circumscribed circle;

- Different physical methods or different software can be used to determine this characteristic;

- This article presents a working methodology, using the Mathcad 15 program, to determine Riley's circularity;

- The working method involves the processing of an image by performing a series of computational to determine the desired characteristic.

\section{REFERENCES}

[1] Herdan, G., Small particle statistics, Ed. Butterworths, London, 1960.

[2] Allen, T., Particle size measurement, Ed. Chapman and Hall, London, 1981.

[3] Barbosa-C'anovas, G.V., Ortega-Rivas, E., Juliano, P., Yan, H., Food powders: physical properties, processing, and functionality, Ed. Kluwer Academic/Plenum Publishers, New York, 2005.

[4] Wadell, H., Sphericity and roundness of rock particles, Journal of Geology, vol. 41, no. 3, 1933, p. $310-331$.

[5] Wentworth, W.C., The shape of rock particle: A discussion, Journal of Geology, vol. 41, 1933, p. 306-309.

[6] Teller, J.T., Equantcy versus sphericity, Sedimentology, vol. 23, 1976, p. 427-728.

[7] Barrett, P.J., The shape of rock particles, a critical review, Sedimentology, vol. 27, 1980, p. 291-303.

[8] Hawkins, A.E., The shape of powder-particle outline, Ed. Wiley, New York, 1993.

[9] Mitchell, J.K., Soga, K., Fundamentals of soil behavior, Third edition, WILEY, 2005.

[10] Arasan, S., Hasiloglu, A.S., Akbulut, S., Shape particle of natural and crashed aggregate using image analysis, International Journal of Civil and Structural Engineering, vol. 1, no. 2, 2010, p. 221-233.

[11] Krumbein, W.C., Pettijohn, F.J., Manual of sedimentary petrography, Ed. Appleton-Century Crofts, Inc., New York, 1938.

[12] Rodriguez, J.M., Edeskär, T., Knutsson, S., Particle shape quantities and measurement techniques-A review, The Electronic journal of geotechnical engineering, 2013, p. 169-198.

[13] Singh, P., Ramakrishnan, P., Powder characterization by particle shape assessment, KONA Powder and Particle Journal, vol. 14, 1996, p. 16-30.

[14] Blott, S.J., Pye, K., Particle shape: a review and new methods of characterization and classification, Sedimentology, vol. 55, no. 1, 2008, p. 31-63.

[15] Rodriguez, J., Edeskär, T., Knutsson, S., Particle shape quantities and measurement techniques: a review, The Electronic journal of geotechnical engineering, vol. 18/A, 2013, p. 169-198. 
[16] Krumbein, W.C., Measurement and geological significance of shape and roundness of sedimentary particles, Journal of Sedimentary Petrology, vol. 11, no. 2, 1941, p. 64-72.

[17] Aschenbrenner, B.C., A new method of expressing particle sphericity, Journal of Sedimentary Petrology, vol. 26, no. 1,1956, p. $15-31$.

[18] Pye, W., Pye, M., Sphericity determination of pebbles and grains, Journal of Sedimentary Petrology, vol. 13, no. 1, 1943, p. 28-34.

[19] Persson, A.L., Image analysis of shape and size of fine aggregates, Engineering Geology, vol. 50, 1998, p. 177-186.

[20] Andersson, T., Estimating particle size distributions based on machine vision, PhD Thesis, University of Technology, Lulea, 2010.

[21] Mo, P.Q., Internal rolling method for particle shape evaluation and reconstruction, PLOS ONE, vol. 15, no. 11, 2020, art. no. e0242162.

[22] Mora, C.F., Kwan, A.K.H., Chan, C.H., Particle size distribution analysis of coarse aggregate using digital image processing, Cement and Concrete Research, vol. 28, 1998, p. 921-932.

[23] Rodriguez, J.M., Importance of the particle shape on mechanical properties of soil materials, $\mathrm{PhD}$ Thesis Universite Tstryckeriet, Lulea, 2013.

[24] https://community.ptc.com/t5/PTC-Mathcad/Fitting-an-Ellipse-to-a-Blob-re-post/td-p/174629 (20.08.2020). 